\title{
Drug Hypersensitivity Reactions in Hospital-Admitted Children: A Single Center Study in Southern Iran
}

\author{
Hesamodin Nabavizadeh, ${ }^{1,2}$ Soheyla Alyasin,,${ }^{1,2}$ Hamidreza Houshmand, ${ }^{2,3,},{ }^{*}$ Gholamreza Houshmand, ${ }^{4}$ \\ Mohammad Bahadoram, ${ }^{5}$ Sara Bayat, ${ }^{6}$ Hossein Esmaeilzadeh, ${ }^{1,2}$ and Mehdi Goudarzi ${ }^{7}$ \\ ${ }^{1}$ Department of Allergy and Clinical Immunology, Shiraz University of Medical Sciences, Shiraz, Iran \\ ${ }^{2}$ Allergy Research Center, Shiraz University of Medical Sciences, Shiraz, Iran \\ ${ }^{3}$ Department of Pediatrics, Division of Allergy and Clinical Immunology, School of Medicine, Urmia University of Medical Sciences, Urmia, Iran \\ ${ }^{4}$ Department of Pharmacology, School of Medicine, Mazandaran University of Medical Sciences, Sari, Iran \\ ${ }^{5}$ Medical Student Research Committee and Social Determinant of Health Research center, Ahvaz Jundishapur University of Medical Sciences, Ahvaz, Iran \\ ${ }^{6}$ Medical Student Research Committee, Mazandaran University of Medical Sciences, Sari, Iran \\ ${ }^{7}$ Medicinal Plant Research Center, Ahvaz Jundishapur University of Medical Sciences, Ahvaz, Iran \\ "Corresponding author: Hamidreza Houshmand, Department of Pediatrics, Division of Immunology and Allergy, Allergic Research Center, Shiraz University of Medica \\ Science, Shiraz, Iran. Tel: +98-9143433913, Fax: +98-7136474293, E-mail: houshmand_ha@sums.ac.ir
}

Received 2016 November 29; Revised 2017 May 24; Accepted 2018 March 10.

\begin{abstract}
Background: Drug allergy is subtype of adverse drug reactions that is mediated by immunologic mechanism. Sever drug allergy is poorly understood in children that leads to hospitalization.

Objectives: The goal of this research was to study severe drug hypersensitivity reactions in hospital-admitted children in southwestern Iran.

Materials and Methods: This is a retrospective study conducted in Namazee hospital, southwestern Iran, the medical records of 33 children with drug allergies during 2011 till 2014 were reviewed.

Results: Drug allergies have mainly been studied in adults. In the current study, 19 patients were females (57.9\%) and 14 were males (42.1\%). Maculopapular rashes were seen in $90.9 \%$ of these patients (30 persons). Overall, drug reactions were seen more commonly in females. The mean age was also higher in females. The highest rate of drug reactions were seen with beta lactams and phenobarbital, including $42.4 \%$ of patients (14 persons) and 39.4\% of patients (13 persons), respectively. Dress syndrome and Steven-Johnson syndrome were the most common reactions in these patients, including $27.3 \%$ ( 9 persons) and $24.2 \%$ of patients ( 8 persons), respectively. Toxic epidermal necrosis was the least common reaction in the patients, involving only $6.1 \%$ of patients (2 persons).

Conclusions: Maculopapular rashes were the most common drug reactions. In the current study, drug rash with eosinophilia and systemic symptoms (DRESS) syndrome was the most common and toxic epidermal necrolysis (TEN) was the least common cause of admission.
\end{abstract}

Keywords: Drug Hypersensitivity Syndrome, Children, Hospital Admission Tests, Stevens Johnson Syndrome, HLA Antigens, Eosinophilia

\section{Background}

Drug allergy has always been a major issue concerning physicians worldwide. Drug reactions are serious and may even cause hospital admissions leading to unwanted expenses for the patient and insurance companies. New drugs have been presented in the 21st century, and their profound use has increased the incidence of drug allergies. Drug reactions can be categorized to 2 major categories: A, predictable adverse reactions for specific drugs (e.g. liver failure at high doses of acetaminophen). B, Unpredictable adverse reactions (e.g. tinnitus in aspirin use) (1). Some of the latter reactions can be attributed to spe- cific HLAs in humans; therefore, the term B adverse reactions might be gradually replaced by genetic causes. In order to describe adverse drug reactions, terms such as immediate and late were used. Immediate drug reactions occur within 1 hour after consumption and are IgE-mediated. In this type, urticaria, angioedema, rhinitis, conjunctivitis, bronchospasm, and gastrointestinal symptoms (nausea, vomiting, and diarrhea), or even anaphylaxis may be seen. Late adverse reactions are seen from 1 hour to days after consumption, presenting as late urticaria, maculopapular rashes, fixed drug eruptions, vasculitis, toxic epidermal necrolysis (TEN), stevens johnsons-syndrome (SJS) (Table 1), drug reaction with eosinophilia and systemic symp- 
tom (DRESS), and acute generalized exanthematous pustulosis (AGEP). In this latter type of drug reaction, visceral organs can be involved without skin involvement, e.g. hepatitis, renal failure, pneumonitis, anemia, neutropenia, and thrombocytopenia. T-cell mediated reactions are responsible for late adverse reactions.

Adverse drug reactions (ADR) have been reported in $10 \%$ to $20 \%$ of hospitalized and $25 \%$ of outpatients. Most adverse reactions are type A, whereas type B was seen in only $10 \%$ to $15 \%(1,2)$. Beta lactams and NSAIDs are responsible for most causes of adverse drug reactions (3). The use of anticonvulsants, radio contrast agents, and neuromuscular blockers has recently shown an increase in ADR (4). Epidemiologic studies have shown that dermatologic reactions, such as maculopapular rashes and urticaria, are the most frequent ADR (5). Severe and fatal reactions, such as TEN, SJS, and DRESS syndrome, are very rare. Pichler et al. described a direct drug reaction named Pi-concept. According to this hypothesis, chemically neutral drugs cannot bind covalently with peptides; hence, they are not able to stimulate the immune system. If the drug is able to connect with HLA molecules of presenting cells or receptors on T cells (HLA B 5701), a very strong T cell response will be induced. In viral diseases, such as EBV, CMV, HIV, HHV-6, and autoimmune diseases, in which the activity of the immune system is increased, the threshold of cellular activity in the reaction to drugs will decrease $(6,7)$. This may be the cause of bizarre reactions, i.e. a hypersensitivity reaction after using a drug without a previous sensitivity phase, or a drug sensitivity associated with viral infections, in which $T$ cell stimulation threshold is decreased and these cells produce a large number of cytokines. T cells will express more MHC on their surface, increasing their response. Some infections may cause an inflammatory reaction resulting in an increased drug allergy; e.g. immunologic skin reactions with sulfonamides in HIV patients $(6,8)$.

\section{Objectives}

The goal of the current research was to study drug hypersensitivity reactions in hospital-admitted children.

\section{Methods}

\subsection{Study Population}

The current researchers studied documents of 33 patients admitted between 2006 and 2014 to a referral hospital in southern Iran, using their medical charts. It was found that the culprit drugs in patients were differentiated by age, gender, drug, side effect, and treatment received. According to DRESS scoring system, patients with a score of
4 or more were included in the current study (7). The exclusion criteria included positive blood cultures, positive hepatitis markers and positive test results for mycoplasma and chlamydia infections. The diagnosis was based on the idea of the physician and the chart (Table1). Serum sickness was mainly caused by drugs and presented with malaise, urticarial, and fever with arthritis, arthralgia, nephritis, and vasculitis. These patients were also included in the current study. Erythema multiform presents with different types of rash-like papules, macules, bullas, vesicles, or plaquelike rashes, which sometimes have pruritus and a burning sensation. Diagnosis is based on the presence of classic target-like lesions. Oral lesions extend to the vermillion border and don't involve the buccal mucosa. These lesions look like a rash but do not disappear within 24 hours. Patients presenting maculopapular rashes following drug consumption without other manifestations were classified as having drug reactions.

\subsection{Statistical Analysis}

The current study was retrospective and T-test and ChiSquare tests were used to compare different groups. A P value of $<0.05$ was considered clinically significant in all cases.

\section{Results}

The results showed that of the 33 patients, 19 were females (57.9\%) with a mean age of $7.41 \pm 3.684$ years old and 14 were males $(42.1 \%)$ with a mean age of $4.58 \pm 3.856$ years. Males were 7 months to 14 years old and females were 22 months to 12 years old. Overall, 17 patients had reactions after using anticonvulsants (51.5\%) and 16 after antibiotics (48.5\%) (Table 2).

Comparing males and females using Chi-square analysis showed no significant difference in cough, abdominal pain, jaundice, conjunctivitis, pruritus, infection with EBV, vesicular lesions, edema, seizure, mucosal, and genitalia involvement. The $P$ values were above 0.05 in all of the above. Hepatomegaly was seen in 3 cases (9.1\%), and 2 had splenomegaly (6.1\%). Hepatomegaly was seen in 3 of the females (15.8\%), and 2 males had splenomegaly (14.3\%), where Chi-square showed no significant difference between the genders ( $P$ value 0.085). Maculopapular rashes were seen in 30 of 33 patients (90.9\%) without a significant difference between the 2 genders ( $\mathrm{P}$ value 0.478 ). Erythematous lesions were seen in 23 patients (69.7\%), 50\% of males and $84.2 \%$ of females, with a P value of 0.087 . The mean temperature was 38.2 degrees Celsius in both males and females. Developmental delay was seen in 4 patients (12.1\%), and it was mainly seen in those receiving anticonvulsants with 


\begin{tabular}{|c|c|c|c|c|c|c|}
\hline Diagnosis & Mucosal Lesions & \multicolumn{5}{|c|}{ Typical Skin Lesions } \\
\hline Stevens Johnsons-syndrome (SJS) & Erosion at $>$ two sites & \multicolumn{5}{|c|}{ Croups of lesions on skin, conjunctiva, mouth and genitalia, detachment of $<10 \%$ of body surface area } \\
\hline Toxic epidermal necrolysis (TEN) & Erosion at $>$ two sites & \multicolumn{5}{|c|}{$\begin{array}{l}\text { Lesions similar to those with SJS, confluent epidermis separate readily with lateral pressure, } \\
\text { detachment of }>30 \% \text { of body surface area }\end{array}$} \\
\hline Adverse Drug Reactions (ADR) & Total, \% & Male & Female & Male Ratio, \% & Female Ratio, \% & PValue \\
\hline Maculopapular rash & 90.9 & 12 & 18 & 85.7 & 94.7 & 0.478 \\
\hline Erythematous rash & 69.7 & 7 & 16 & 50.0 & 84.2 & 0.087 \\
\hline Itching & 42.4 & 4 & 10 & 28.6 & 52.6 & 0.167 \\
\hline Mucositis & 33.3 & 3 & 8 & 21.4 & 42.1 & 0.213 \\
\hline Conjunctivitis & 24.2 & 2 & 6 & 14.3 & 31.6 & 0.252 \\
\hline Cough & 21.2 & 2 & 5 & 14.3 & 26.8 & 0.403 \\
\hline Abdominal pain & 15.2 & 2 & 3 & 14.3 & 15.8 & 0.905 \\
\hline Genital involvement & 12.1 & 1 & 3 & 7.1 & 15.8 & 0.452 \\
\hline Vesicular rash & 12.1 & 1 & 3 & 7.1 & 15.8 & 0.452 \\
\hline Icterus & 9.1 & 0 & 3 & 0 & 15.8 & 0.119 \\
\hline Hepatomegaly & 9.1 & 0 & 3 & 0 & 15.8 & 0.085 \\
\hline EBV infection & 6.1 & 1 & 1 & 7.1 & 5.6 & 0.854 \\
\hline Erythema multiform & 6.1 & 1 & 1 & 7.1 & 5.6 & - \\
\hline Splenomegaly & 6.1 & 2 & 0 & 7.1 & 0 & - \\
\hline
\end{tabular}

drug side effects. Six of these patients were male (42.9\%) and 11 female (57.9\%). Table 3 shows age, temperature, and laboratory data of the patients.

Table 4 reveals culprit drugs and the number of patients with different drug reactions and their prevalence. Maculopapular rash was seen in $90.9 \%$, DRESS syndrome in $27.3 \%$, SJS in $24.2 \%$, and maculopapular rashes were seen in $90.9 \%$ of the patients. Furthermore, TEN was seen in $6.1 \%$ of the patients. Prednisolone with a dose of $1 \mathrm{mg} / \mathrm{kg}$ was used as treatment in 21 patients (63.6\%) and IVIG in 8 patients (24.2\%). Hydroxyzine and cetirizine were also used in some patients. Only 1 patient died of DRESS syndrome (3\%). Personal and family history for drug allergies was an important factor in drug reactions of children.

\section{Discussion}

Adverse drug reactions (ADR) as a cause of admission to hospital can happen in variable degree and with most of drugs $(9,10)$. In the current study, drug allergy was seen in females $(57.9 \%)$ more than males (42.1\%) and the mean age was higher in females. A systematic study of 76 patients with adverse drug reactions showed that it was mostly seen between birth and 4 years of age (10). Accord- ing to a previous study, drug allergy was seen in females more than males, and the mean age was higher in females. This might have been due to the low number of patients studied. Beta-lactams and phenobarbital were the drugs that caused the most adverse reactions (Table 4). Turk et al. reported a $2 \%$ to $3 \%$ incidence of cutaneous adverse drug reactions (CADR), and antibiotics, NSAIDs, and anticonvulsants were the most common adverse reactions (11). The most common CADR was morbilliform or maculopapular exanthema. These CADR were seen more commonly in females. The higher incidence of drug reactions in females may be attributed to the fact that their immune systems were more sensitive to stimulations, just as autoimmune diseases. Fernandez et al. revealed that beta-lactams and cephalosporins were the main antibiotics with drug reactions. They also showed a cross-reaction between these 2 drugs due to their side chains (12). In the current study, phenobarbital was the anticonvulsant with the most drug reactions. Sodium valproate and lamotrigine demonstrated the least reactions with a 3-\% rate in this group (Table 4). In a descriptive study conducted by Karimzadeh et al., children under 4 years of age were studied regarding adverse reactions with antiepileptic drugs in 2010 to 2012. Most reactions were maculopapular and the main culprit 


\begin{tabular}{|c|c|c|c|c|}
\hline Parameter & No. & Minimum & Maximum & Mean \pm SD \\
\hline Age, $y$ & 33 & 1 & 14 & $6.21 \pm 3.962$ \\
\hline Temperature, ${ }^{\circ} \mathrm{C}$ & 33 & 37 & 42 & $38.20 \pm 1.107$ \\
\hline ESR & 32 & 1 & 90 & $30.66 \pm 32.369$ \\
\hline WBC count & 32 & 2500 & 27700 & $9696.88 \pm 6044.431$ \\
\hline PLT count & 32 & 24000 & 546000 & $297156.52 \pm 148730.39$ \\
\hline Eosinophilia,\% & 13 & 1 & 42 & $8.85 \pm 13.613$ \\
\hline
\end{tabular}

\begin{tabular}{|c|c|c|c|c|c|c|c|c|c|}
\hline Culprit Drug & Male and Female & Male & Female & DRESS & SJS & $\begin{array}{l}\text { Erythema } \\
\text { Multiform }\end{array}$ & Serum Sickness & TEN & Drug Reaction \\
\hline Phenobarbital & $13(39.4)$ & 7 & 6 & 9 & 1 & - & - & - & 3 \\
\hline Carbamazepine & $2(6.1)$ & - & 2 & - & 2 & - & - & - & - \\
\hline $\begin{array}{l}\text { Beta lactams and } \\
\text { cephalosporin }\end{array}$ & $14(42.1)$ & 7 & 7 & - & 4 & 2 & 3 & 2 & 3 \\
\hline Cotrimoxazole & $2(6.1)$ & 1 & 1 & - & - & 1 & - & - & 1 \\
\hline Lamotrigine & $1(3.0)$ & - & 1 & - & - & - & - & - & 1 \\
\hline Sodium valproate & $1(3.0)$ & - & 1 & - & 1 & - & - & - & - \\
\hline Total & $33(100)$ & 15 & 18 & $9(27.3)$ & $8(24.2)$ & $3(9.1)$ & $3(9.1)$ & $2(6.1)$ & $8(24.2)$ \\
\hline
\end{tabular}

Abbreviations: DRESS, Drug Reaction with Eosinophilia and Systemic Symptom; TEN, Toxic Epidermal Necrolysis; SJS, Stevens Johnsons-Syndrome.

${ }^{a}$ Values are expressed as No. (\%).

drug was phenobarbital (70\%), whereas the least important was lamotrigine (1.4\%). Reactions were seen in aromatic drugs more than non-aromatic ones. It is probable that high consumption and low price made phenobarbital the most common cause of drug reactions (13). Maculopapular lesions were the most common reaction seen in the current study (90.9\%). Systemic reactions had no significant difference between males and females. Developmental delay was seen in 4 patients (12.1\%), mainly in patients receiving antiepileptic drugs. One of the patients had head trauma due to a car accident and received carbamazepine causing SJS. Head trauma is a risk factor for drug reactions related to antiepileptics. Systemic adverse drug reactions were seen more in females (Table 2). Langerova et al. conducted a study in Czechoslovakia on 2093 admitted patients; $2.2 \%$ had ADR. Anti-cancer agents (35\%) and antibiotics (18\%) had the most reactions (14). In this study, female gender and having an oncologic cancer were the risk factors for ADR. Kourouma et al. conducted a retrospective study of 10 years on African children with a diagnosis of SJS and TEN, found in 63\% and 33.3\%, respectively. Sulfonamides had the highest incidence and 3 children died of TEN (15). In the patients of the current study, the most common causes of admission were DRESS syndrome, SJS, and maculopapular reaction; TEN is a rare syndrome. Prednisolone with a dose of $1 \mathrm{mg} / \mathrm{kg}$ was used as treatment in 21 patients (63.6\%) and IVIG in 8 patients (24.2\%). Hydroxyzine and cetirizine were also used in some patients. Overall, 32 out of 33 patients showed a good response to treatment (97\%) and all their symptoms had resolved. Only one patient died due to liver failure with DRESS syndrome. In another study, cutaneous adverse drug reactions (CADR) were studied in 122 patients. The most prevalent ones were urticaria and angioedema. The most common drug involved in CADR was antibiotics (16). In the current study, a 5-yearold male had HIV and tuberculosis and other family members also had HIV. He developed skin lesions after starting cotrimoxazole. Immunologic skin reactions with sulfonamides can be seen in HIV patients. If the current study had more cases, a closer distribution to the population would have been achieved. Patients with drug reactions, who did not need admission were not included in the current study. Limitation of this study is low number of cases. Genetic basis is also probable etiology for higher rate of severe drug allergy in susceptible patients and evaluating specific human leukocyte antigens (HLAs) in these patients might be helpful $(17,18)$. Underlying disease such as immunodeficiency can be a possible etiology for overuse of antibiotics that leads to increase risk of drug allergy $(19,20)$. 


\subsection{Conclusion}

Beta-lactams and phenobarbital were the most culprit drugs causing adverse reactions. Maculopapular rashes were the most common of drug reactions. In our study, DRESS syndrome was the most and TEN the least cause of admission in our patients.

\section{Acknowledgments}

The authors would like to thank Shiraz University of Medical Sciences, Shiraz, Iran and also the center for development of clinical research and statistical group of Nemazee hospital, especially Dr. Hosseini and the personnel of Shahid Motahari clinic for their assistance. The authors would also like to thank Shiraz University of Medical Sciences, Shiraz, Iran and also the center for development of clinical research of Nemazee hospital and Dr. Nasrin Shokrpour for her editorial assistance.

\section{Footnote}

Conflict of Interest: All authors declare no conflict of interest related to the present work.

\section{References}

1. Adkinson Jr NF, Bochner BS, Burks AW, Busse WW, Holgate ST, Lemanske Jr RF. Middleton's allergy: principles and practice. Elsevier Health Sciences; 2013. p. 1274-95.

2. Chiriac AM, Demoly P. Drug allergy diagnosis. Immunol Allergy Clin North Am. 2014;34(3):461-71. vii. doi: 10.1016/j.iac.2014.03.002. [PubMed: 25017672].

3. Dona I, Barrionuevo E, Blanca-Lopez N, Torres MJ, Fernandez TD, Mayorga $\mathrm{C}$, et al. Trends in hypersensitivity drug reactions: more drugs, more response patterns, more heterogeneity. JInvestig Allergol Clin Immunol. 2014;24(3):143-53. quiz 1 p following 153. [PubMed: 25011351].

4. Khan DA, Solensky R. Drug allergy. J Allergy Clin Immunol. 2010;125(2 Suppl 2):S126-37. doi:10.1016/j.jaci.2009.10.028. [PubMed: 20176256].

5. Levi N, Bastuji-Garin S, Mockenhaupt M, Roujeau JC, Flahault A, Kelly JP, et al. Medications as risk factors of Stevens-Johnson syndrome and toxic epidermal necrolysis in children: a pooled analysis. Pediatrics. 20092;123(2 Suppl 2). e297-304. doi:10.1542/peds.2008-1923. [PubMed: 19153164].

6. Pichler WJ. The p-i Concept: Pharmacological Interaction of Drugs With Immune Receptors. World Allergy Organ J. 2008;1(6):96-102. doi: 10.1097/WOX.ob013e3181778282. [PubMed: 23282405]. [PubMed Central: PMC3651037].

7. Kardaun SH, Sidoroff A, Valeyrie-Allanore L, Halevy S, Davidovici BB, Mockenhaupt M, et al. Variability in the clinical pattern of cutaneous side-effects of drugs with systemic symptoms: does a DRESS syndrome really exist? BrJ Dermatol. 2007;156(3):609-11. doi:10.1111/j.13652133.2006.07704.x. [PubMed: 17300272].
8. Cano FG, Rozenfeld S. Adverse drug events in hospitals: a systematic review. Cad Saude Publica. 2009;25 Suppl 3:S360-72. doi: 10.1590/S0102-311X2009001500003. [PubMed: 20027385].

9. Pirmohamed M, James S, Meakin S, Green C, Scott AK, Walley TJ, et al. Adverse drug reactions as cause of admission to hospital: prospective analysis of 18820 patients. BMJ. 2004;329(7456):15-9. doi: 10.1136/bmj.329.7456.15. [PubMed: 15231615]. [PubMed Central: PMC443443].

10. Marrs T, Fox AT, Lack G, du Toit G. The diagnosis and management of antibiotic allergy in children: Systematic review to inform a contemporary approach. Arch Dis Child. 2015;100(6):583-8. doi: 10.1136/archdischild-2014-306280. [PubMed: 25527519].

11. Turk BG, Gunaydin A, Ertam I, Ozturk G. Adverse cutaneous drug reactions among hospitalized patients: five year surveillance. Cutan Ocul Toxicol. 2013;32(1):41-5. doi: 10.3109/15569527.2012.702837. [PubMed: 22812902].

12. Fernandez TD, Mayorga C, Ariza A, Corzo JL, Torres MJ. Allergic reactions to antibiotics in children. Curr Opin Allergy Clin Immunol. 2014;14(4):278-85. doi: 10.1097/ACI.0000000000000069. [PubMed: 24848090].

13. Karimzadeh P, Bakrani V. Antiepileptic drug-related adverse reactions and factors influencing these reactions. Iran J Child Neurol. 2013;7(3):25-9. [PubMed: 24665302]. [PubMed Central: PMC3943074].

14. Langerova P, Vrtal J, Urbanek K. Adverse drug reactions causing hospital admissions in childhood: a prospective, observational, single-centre study. Basic Clin Pharmacol Toxicol. 2014;115(6):560-4. doi: 10.1111/bcpt.12264. [PubMed: 24810357].

15. Kourouma HS, Kouassi YI, Diabate A, Sangare A, Ecra EJ, Kaloga M, et al. [Clinical-epidemiological profile of severe cutaneous drug reactions in children in Abidjan (Cote d'Ivoire)]. Bull Soc Pathol Exot. 2014;107(3):142-5. doi: 10.1007/s13149-014-0360-y. [PubMed: 24792460].

16. Dilek N, Ozkol HU, Akbas A, Kilinc F, Dilek AR, Saral Y, et al. Cutaneous drug reactions in children: a multicentric study. Postepy Dermatol Alergol. 2014;31(6):368-71. doi: 10.5114/pdia.2014.43881. [PubMed: 25610351]. [PubMed Central: PMC4293388].

17. Esmaeilzadeh $\mathrm{H}$, Nabavi M, Aryan Z, Amirzargar AA. Pharmacogenetic tests to predict the efficacy of aspirin desensitization in patients with aspirin-exacerbated respiratory diseases; HLA-DQB302. Expert Rev Respir Med. 2015 10;9(5):511-8. doi: 10.1586/17476348.2015.1081062. [PubMed: 26366802].

18. Mortazavi N, Esmaeilzadeh $\mathrm{H}$, Abbasinazari M, Babaie D, Alyasin S, Nabavizadeh $\mathrm{H}$, et al. Clinical and Immunological Efficacy of Aspirin Desensitization in Nasal Polyp Patients with Aspirin-Exacerbated Respiratory Disease. Iran J Pharm Res. 2017;16(4):1639-47. [PubMed: 29552073]. [PubMed Central: PMC5843326].

19. Nabavi M, Arshi S, Bemanian MH, Aghamohammadi A, Mansouri $\mathrm{D}$, Hedayat $\mathrm{M}$, et al. Long-term follow-up of ninety eight Iranian patients with primary immune deficiency in a single tertiary centre. Allergol Immunopathol (Madr). 2016;44(4):322-30. doi: 10.1016/j.aller.2015.09.006. [PubMed: 26803694].

20. Nabavi M, Arshi S, Bemanian MH, Shakeri R, Taghvaei B, Ghalebaghi B, et al. Long-term follow-up of ninety-eight Iranian patients with primary immune deficiency in a single tertiary centre. Allergol Immunopathol. 2016;44(4):322-30. doi: 10.1016/j.aller.2015.09.006. [PubMed: 26803694]. 